\title{
Breast Cancer Distribution Pattern in a Pathology Center in South East Nigeria
}

\author{
Samuel I Ogenyi ${ }^{1 *}$, Nancy C Ibeh ${ }^{1}$, June A Onu ${ }^{1}$, Jonathan U Madukwe ${ }^{2}$, Onyekachi A Onu ${ }^{3}$ and Felix E Menkiti ${ }^{4}$ \\ ${ }^{1}$ Department of Medical Laboratory Science, Faculty of Health Sciences and Technology, College of Health Sciences, Nnamdi Azikiwe \\ University, Nnewi Campus, Nigeria \\ ${ }^{2}$ Department of Histopathology, National Hospital Abuja, Nigeria \\ ${ }^{3}$ Department of Surgery, Nnamdi Azikiwe University Teaching Hospital, Nnewi, Anambra State, Nigeria \\ ${ }^{4}$ Department of Anatomic and Forensic Medicine, Faculty of Medicine, College of Health Sciences, Nnamdi Azikiwe University, Nnewi \\ Campus, Nigeria \\ *Corresponding Author: Samuel I Ogenyi, Department of Medical Laboratory Science, Faculty of Health Sciences and Technology, \\ College of Health Sciences, Nnamdi Azikiwe University, Nnewi Campus, Nigeria.
}

Received: December 7, 2021; Published: January 13, 2022

\section{Abstract \\ Background}

Breast cancer remains the most commonly diagnosed cancer in women accounting for about $11 \%$ of all cancer types diagnosed globally annually and one of the leading causes of women mortality in spite of significant improvements in treatment. Treatment of breast cancer in Nigeria is based of expression pattern of ER, PR and HER2 using targeted therapies.

\section{Methods}

Two hundred and seventy three (273) archived paraffin wax processed breast tissue samples were sorted and selected from the histopathology laboratories and museums of the facility. Data were realized from clinical records, operation notes and histopathology results of the patients involved. HER2, ER and PR expressions were assessed by immunohistochemistry (Avidin-biotin complex method). Expression patterns were scored based on proportion and intensity of immune-labeling using semi-quantitative method.

Results

The age of patients ranged from 30 to 75 years with greater population falling between 30-45 years. Numerically, breast cancer cases seen increased progressively from 2015 to 2019. Most breast cancer tissues were ER positive 146 (53.5\%) while 65 (23.8\%) samples were HER2 positive. A greater percentage of the samples assessed were ER/PR/HER positive (Triple positive) while ER negative/PR positive accounted for the least case.

\section{Conclusion}

The present study revealed a progressive increase of breast cancer incidence from the year 2015 to 2019 in the centre studied with ER/PR positive cancers being the most prevalent followed by triple negative cancers. More awareness to breast cancer risk factors, prevention, screening and early presentation to hospitals should be intensified with emphasis on early detection and targeted and personalized management.

Keywords: Breast Cancer; ER; PR; HER2

\section{Introduction}

Breast cancer is the most commonly diagnosed cancer in women [1], accounting for about 11\% of all cancer types diagnosed globally annually and a constituent of the leading causes of women mortality in spite of significant improvements in treatment [2]. Studies re- 
vealed that screening and early detection through breast self-examination, mammography and clinical breast examination can reduce breast cancer mortality rate [3]. Earlier study by Al-Naggar and Bobryshev [4] however, identified poor interactions with doctors and the screening procedure itself (such as pain and discomfort) as barriers to breast cancer screening. Several breast cancer risk factors such as family history, menarche, parity, age, menopausal status, age at first live birth, genetic mutations, breast feeding, etc. [5], and lifestyle factors such as fatty diet and lack of exercise are well known [6]. Based on Estrogen Receptor (ER) status, Progesterone Receptor (PR) status and Human Epidermal Growth-factor Receptor-2 (HER-2/neu) expression, breast cancers are categorized into one of five major subtypes to facilitate targeted therapy and personalized management $[7,8]$. The subtypes are: luminal A [PR+ and/ or ER+, HER-2/neu-], luminal B (PR+ and/or ER+, HER-2/neu+), HER-2/neu over expressing (PR-, ER-, HER-2/neu+), basal-like (PR-, ER-, HER-2/neu-, cytokeratin 5/6+ and/or epidermal growth factor receptor+) and normal breast-liketumours [9]. In a more practical clinical application, breast cancers can be categorized and described as with the following terms: triple negative, triple positive, bi positive and mono positive breast cancers.

The human epidermal growth factor receptor 2 (HER2) is a $185 \mathrm{kDa}$ transmembrane tyrosine kinase receptor encoded by the ERBB2 breast cancer oncogene [10] (King et al., 1985). Its over expression leads to constitutive activation of mitogen-activated protein kinase (MAPK) and protein kinase B (AKT) signaling pathways which result in elevated metabolic function, increase in proliferation and enhanced tumour invasiveness [11]. In breast cancer cells, HER2 over expression leads to PI3K/AKT pathway activation [12]. The activation and downstream signaling of AKT have been shown to inhibit the arrest of cell cycle and block trastuzumab-mediated apoptosis [13]. Breast cancer cells with high levels of HER2 expression has frequently been found to be associated with tumours that are more agressive and have poor sensitivity to standard chemotherapeutic agents [14]. The over expression of HER2 oncogene has been implicated in the growth and progression of some violent breast cancer types. This protein serves as an important biomarker and enhances targeted therapy for about 30\% of breast cancer population [15]. The Human epidermal growth factor receptor family does not only comprise of HER2 protein, but includes as well HER1, HER3 (ErbB3), and HER4 (ErbB4) [16]. The HER2 protein dimerizes with HER1, HER3, or HER4 [16]. HER2 does not have a known ligand, and this permits it to always be in open confirmation to dimerize with other members of EGFR family [17]. In essence, when there is an over expression of the HER2 gene, it gives room for cell growth, cell differentiation and survival through a complex pathway enhanced by the activation of PI3K/Akt as well as the Ras/Raf/MEK/MAPK pathways [18]. Present on the surface of HER2-positive cancer cells are about two millions HER2 proteins. These are about 100 times more than what is found on a normal cell [19]. HER2 dimerization is an important step in the signaling pathway that results in cancer development [20]. Breast cancers that over express HER2 are mostly aggressive form of the disease and about one in five women (20\% of women) found to be with breast cancer worldwide will be positive for HER2 type [21].

ER, which is a factor in transcription and a protein of the nuclear receptor family, regulates transcription of several genes, leading to division of cells. It plays vital role in the development of the mammary gland and cell growth during pregnancy. ER serves as a key factor in the tumourigenesis of ER positive (ER+) breast cancer, where it is involved in the initiation of overwhelming cell division, leading to tumour onset and progression. Endocrine treatment that targets ER are usually employed in the treatment of ER+ breast cancers [22]. PR similarly belongs to the super family of steroid receptors that regulates progesterone in its target tissues. PR expression is restricted to the luminal epithelial cells in mammary gland. Breast cancer cells that are progesterone positive (PR+) have receptors, which permit them to utilize the hormone in their growth and invasion and treatment is targeted at blocking PR secretion. Co expression of ER and PR in breast cancers are common while only about 1\% of cases have PR expression without expressing ER [23].

This study is aimed at the assessment and characterization of breast cancers types in a tertiary health institution in Nigeria over a given period. This will reveal the overall prevalence and serve as benchmark for evaluation of current management strategies.

\section{Materials and Method}

Study area/Study design

This was a retrospective cross sectional study using archived breast tissue samples collected from a tertiary healthcare institution 
in the South East Nigeria within the period of 2015 to 2019.

\section{Ethical approval}

Ethical approval for this study was sought and obtained from the Ethics Committee of Nnamdi Azikiwe University Teaching Hospital (NAUTH) Nnewi(NAUTH/CS/66/VOL.12/224/2019/087).

\section{Sample collection}

Two hundred and seventy three (273) archived paraffin wax processed breast tissue blocks were sorted and picked from the histopathology Laboratories and museums of the facility. Data were obtained from clinical records, operation notes and histopathology reports of the subjects.

\section{Tissue preparation}

Tissue blocks were re-embedded in fresh paraffin wax, $3 \mu$ thick sections cut with the aid of a rotary microtome (HM340E Thermo Scientific. Massachusetts, United States of America)., cut sections floated out on a lukewarm Leica water bath, mounted on slides previously coated with poly-l-lysine, drained, labelled and placed on Leica hot plate in order to dry and affix the tissue onto the slides.

\section{Staining}

Cut Sections were stained by Haematoxylin and Eosin (H\&E) method and morphological diagnosis of each sample confirmed before proceeding to ER, PR and HER2 immunostaining.

\section{Immunohistochemical Staining (IHC)}

IHC of test materials and positive controls were carried following the method used by Nishio et al [24]. Monoclonal antibodies for ER, PR and HER2 were employed for immunohistochemistry. Exposed Mouse and Rabbit Specific horseradish peroxidase/diaminobenzidine (HRP/DAB) detection IHC kit was used for immunostaining while detection of immunoreactivity was performed according to manufacturer's instruction. The antibodies together with the detection kits were obtained from Abcam Plc Cambridge UK through Biotec Limited.

\section{Immunoreactivity Scoring}

Immunoreactivity was scored semi-quantitatively according to Zlobec et al [25]. This was based on percentage of positively stained cells (area) and the staining intensity (strong, moderate, weak). A score of $5+$ was assigned to $\geq 80 \%$ of epithelial and/ or stromal cells staining positive with strong intensity, $4+$ was given to $\geq 50 \%$ (but $<80 \%$ ) of epithelial cells and/ stromal cells staining positive with strong intensity or $\geq 80 \%$ of cells staining positive with moderate to weak intensity; $3+$ was assigned to $\geq 30 \%$ of epithelial and/ or stromal cells staining positive with strong intensity or $\geq 50 \%$ (but $<80 \%$ ) of cells staining positive with moderate to weak intensity; $2+$ was assigned to $\geq 10 \%$ of cells staining positive with strong intensity or $\geq 30 \%$ ( but $<50 \%$ ) of cells staining moderate to weak intensity and $1+$ was assigned to $\geq 10 \%$ of cells (but $<30 \%$ of positive cells) staining positive with moderate to weak intensity; 0 was assigned to $<10 \%$ of positivity irrespective of the intensity of staining.

\section{Data analyses}

Numerical data were summarized using mean and standard deviation, whereas categorical data were presented using frequency and percentages. Immunoreactivity pattern was expressed in percentages.

\section{Results and Discussion}

The age of subjects ranged from 30 to 75 years with greater population falling between 30-45 years. Numerically, breast cancer cases 
Breast Cancer Distribution Pattern in a Pathology Center in South East Nigeria

seen increased progressively from 2015 to 2019. Most breast cancer tissues were ER positive 146 (53.5\%) while 65 (23.8\%) samples were HER2 positive (Table 1). A greater percentage of the samples assessed were ER/PR/HER positive (Triple positive) while ER negative/PR positive accounted for the least case (Figure 1). Photomicrographs of immunoreactivitive patterns were shown in plate 1.

\begin{tabular}{|l|c|c|c|c|c|c|c|}
\hline \multirow{2}{*}{ Year } & \multirow{2}{*}{$\begin{array}{c}\text { Number of } \\
\text { samples (n) }\end{array}$} & \multicolumn{2}{|c|}{ ER } & \multicolumn{2}{c|}{ PR } & \multicolumn{2}{c|}{ HER2 } \\
\cline { 3 - 8 } & $37(13.6 \%)$ & $19(51.4 \%)$ & $18(48.6 \%)$ & $14(37.8 \%)$ & $23(62.2 \%)$ & $9(24.3 \%)$ & $28(75.7 \%)$ \\
\hline 2015 & $49(17.9 \%)$ & $26(53.1 \%)$ & $23(46.9 \%)$ & $19(38.8 \%)$ & $30(61.2 \%)$ & $12(24.5 \%)$ & $37(75.5 \%)$ \\
\hline 2016 & $54(19.8 \%)$ & $29(52.7 \%)$ & $25(47.3 \%)$ & $22(40.7 \%)$ & $32(59.3 \%)$ & $13(24.1 \%)$ & $41(75.9 \%)$ \\
\hline 2017 & $61(22.3 \%)$ & $33(54.1 \%)$ & $38(45.9 \%)$ & $24(39.3 \%)$ & $37(60.7 \%)$ & $14(22.9 \%)$ & $47(77.1 \%)$ \\
\hline 2018 & $72(26.4 \%)$ & $39(54.2 \%)$ & $33(45.8 \%)$ & $29(40.3 \%)$ & $43(59.7 \%)$ & $17(23.6 \%)$ & $55(76.4 \%)$ \\
\hline 2019 & $273(100 \%)$ & $146(53.5 \%)$ & $127(46.5 \%)$ & $108(39.6 \%)$ & $165(60.4 \%)$ & $65(23.8 \%)$ & $207(76.2 \%)$ \\
\hline Total (N) & & &
\end{tabular}

Table 1: Characterization of breast according to years.

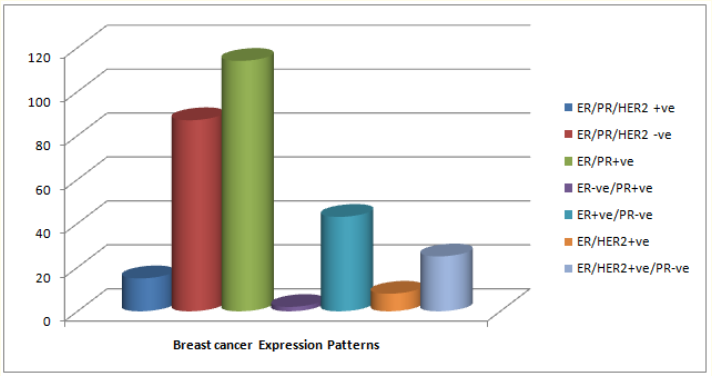

Figure 1: Co-Expression pattern of ER, PR and HER2.

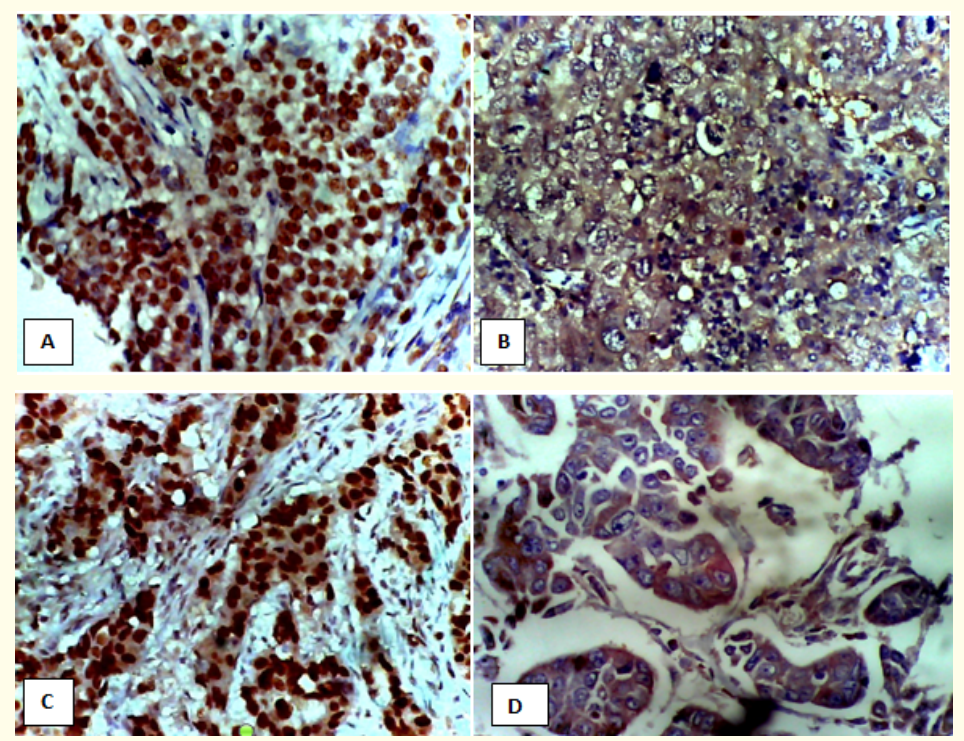

Citation: Samuel I Ogenyi., et al. “Breast Cancer Distribution Pattern in a Pathology Center in South East Nigeria”. Medicon Medical Sciences 2.2 (2022): 11-17. 


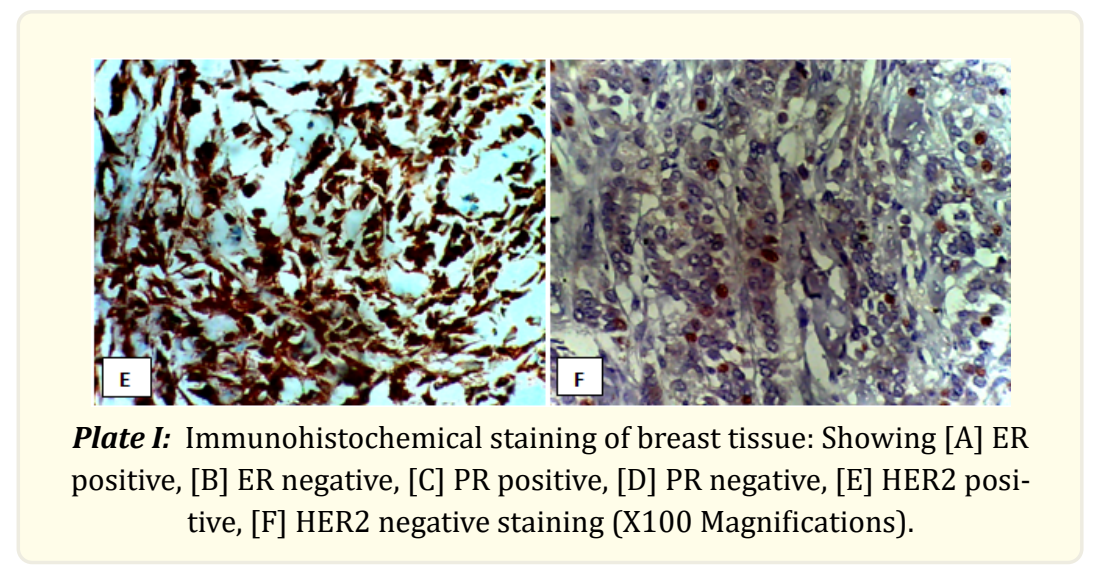

Breast cancer is the most commonly diagnosed cancer in women [1], accounting for about $11 \%$ of the different cancer types diagnosed globally annually and a leading cause of women mortality despite significant improvements in treatment [2]. Breast cancer treatment in Nigeria is based on the expression pattern of ER, PR and HER2. Targeted therapy for ER, PR and HER2 are readily available for treatment and follow up after immunohistochemical staining.

The current study reported progressive increase in incidence from 2015 to 2019. This agrees with the findings of Olaogun et al [26] (who reported increased breast cancer incidence in Nigeria from 15.3 per 100,000 in 1976 to 64.6 per 100,000 in 2012) and Azubike et al [27]. The increased number of presented cases may be connected to increased awareness of the disease burden among the sub-urban population. This may not even reflect the actual incidence, as majority of patients are uninformed and may not present for treatment. The increased incidence may as well be linked to adoption of lifestyles, which predisposes to the development of the disease. Lifestyle factors, such as increase in the use of breast implants, breast enlargement pills; to mention but a few, which hitherto uncommon in our clime have become popular practices. Estrogen receptor expressing tumours accounted for greater percentage of all presented cases, with ER/PR positive (bi positive) cancers taking the lead followed by triple negative cases. This corroborates partly with an earlier study by Oboma and Madukwe [23]. Evaluation of breast cancer hormonal status forms the mainstay to the treatment of the disease and gives an insight to individual cancer behavior. Bi positive cancers are usually very aggressive with inclination for metastasis. Triple negative cancers similarly, are very aggressive and pose many challenges to management. There has not been a uniformly accepted targeted therapy for this category of cancer.

The age distribution of patients reported in this study ranges from third to seventh decades of life. This is in consonance with the findings of Olaogun et al [26]. This study however, reported increased incidence amongst patients within the third decade of life. This seems like a new dimension to the disease and calls for more study.

\section{Conclusion}

The present study revealed a progressive increase in the incidence of breast cancer from 2015 to 2019 in the centre studied. Subjects within the third decade of life constitute the greater percentage of the population. The breast cancer type mostly presented is ER/ PR positive followed by triple negative cancers. More awareness to the risk factors of breast cancer, screening, prevention, and early presentation to hospitals should be intensified. More emphasis should be targeted towards early diagnosis, targeted and personalized management.

\section{Acknowledgements}

The authors express their immense gratitude to the staff and management of Histopathology department, Nnamdi Azikiwe Univer-

Citation: Samuel I Ogenyi., et al. "Breast Cancer Distribution Pattern in a Pathology Center in South East Nigeria". Medicon Medical Sciences 2.2

(2022): 11-17. 
sity Teaching Hospital Nnewi for granting us the approval to go ahead with the study and to Professor C. O. Akosile for proof reading the manuscript.

\section{References}

1. Siegel R., et al. Cancer statistics, 2014. Cancer 64.1 (2014): 9-29.

2. Majeed W., et al. "Breast cancer: major risk factors and recent developments in treatment". Asian Pacific Journal of Cancer Prevention 15.18 (2014): 3353-3358.

3. Fouladi N., et al. "Beliefs and behaviors of breast cancer screening in women referring to health care centers in northwest Iran according to the champion health belief model scale". Asian Pacific Journal of Cancer Prevention 14.11 (2013): 6857-6862.

4. Al-Naggar R., et al. "Practice and barriers of mammography among Malaysian women in the general population". Asian Pacific Journal of Cancer Prevention 13.8 (2012): 3595-3600.

5. Gürdal S., et al. "The Effects of Educational Level on Breast Cancer Awareness: A Cross Sectional Study in Turkey". Asian Pacific Journal of Cancer Prevention 13.1 (2012): 295-300.

6. Berkiten A., et al. "Meta analysis of studies about breast self examination between 2000-2009 in Turkey". Asian Pacific Journal of Cancer Prevention 13.7 (2012): 3389-3397.

7. Zubeda S., et al. A. "HER-2/neu status: a neglected marker of prognostication and management of breast cancer patients in India”. Asian Pacific Journal of Cancer Prevention 14.4 (2013): 2231-2235.

8. Qi Y., et al. "Ultrasonographic features of triple-negative breast cancer: a comparison with other breast cancer subtype". Asian Pacific Journal of Cancer Prevention 16.8 (2015): 3229-3232.

9. Boyle P. “Triple-negative breast cancer: epidemiological considerations and recommendations”. Annals ofOncology 23 (2012): 7-12.

10. Schettini F., et al. "Hormone Receptor/Human Epidermal Growth Factor Receptor 2-positive breast cancer: Where we are now and where we are going". Cancer Treatment Reviews 46 (2016): 20-26.

11. King C., et al. "Amplification of a novel v-erbB-related gene in a human mammary carcinoma”. Science 229.4717 (1985): $974-976$.

12. Hynes N., et al. "PI3K inhibition overcomes trastuzumab resistance: blockade of ErbB2/ErbB3 is not always enough". Cancer Cell 15.5 (2009): 353-355.

13. Yakes F., et al. "Herceptin-induced inhibition of phosphatidylinositol-3 kinase and Akt Is required for antibody-mediated effects on p27, cyclin D1, and antitumor action". Cancer Research 62.14 (2002): 4132-4141.

14. Martin M and López S. "Emerging Therapeutic Options for HER2-Positive Breast Cancer". American Society of Clinical Oncology-Educational Book 35 (2016): 64-70.

15. Mitri Z., et al. "The HER2 Receptor in Breast Cancer: Pathophysiology, Clinical Use, and New Advances in Therapy”. Chemotherapy Research and Practice (2012): 1-7.

16. Bailey T., et al. "Mechanisms of Trastuzumab resistance in ErbB2-driven breast cancer and newer opportunities to overcome therapy resistance". Journal of Carcinogenesis 10 (2011): 317-328.

17. Graus-Porta D., et al. "ErbB-2, the preferred heterodimerization partner of all ErbB receptors, is a mediator of lateral signaling". The EMBO Journal 16.7 (1997): 1647-1655.

18. Arteaga C., et al. "Treatment of HER2-positive breast cancer: current status and future perspectives". Nature Reviews Clinical Oncology 9.1 (2011): 16-32.

19. Sautter G., et al. "Guidelines for human epidermal growth factor receptor 2 testing: biologic and methodologic considerations". Journal of Clinical Oncology 27.8 (2012): 1323-1333.

20. Lewis P., et al. "Targeting HER2-positive breast cancer with trastuzumab-DM1,an antibody-cytotoxic drugconjugate”. Cancer Research 68.22 (2008): 9280-9290.

21. Wolff A., et al. "American society of clinical oncology/college of American pathologists clinical practice guideline update: Recom- 
mendations for human epidermal growth factor receptor 2 testing in breast cancer". Journal of Clinical Oncology 31.31 (2013): 3997-4013.

22. Subik K., et al. "The expression patterns of ER, PR, HER2, CK5/6, EGFR, Ki-67 and AR by immunohistochemical analysis in breast cancer cell lines". Breast Cancer 4 (2010): 35-41.

23. Madukwe UAJ and Oboma YI. "Triple Negative Breast Cancer in a Private Immunohistochemistry Laboratory in Abuja Nigeria". Advances in Biological Research 10.1 (2016): 58-64.

24. Nishio K., et al. Cancer Science 97.7 (2013): 642-648.

25. Zlobec I., et al. "Selecting immunohistochemical cut-off scores for novel biomarkers of progression and survival in colorectal cancer". Journal of clinical pathology 60.10 (2006): 1112-1116.

26. Olaogun JG., et al. "Socio-demographic, pattern of presentation and management outcome of breast cancer in a semi-urban tertiary health institution". Pan African Medical Journal 36 (2020): 363-373.

27. Azubuike SO., et al. "Rising global burden of breast cancer: the case of sub-Saharan Africa (with emphasis on Nigeria) and implications for regional development: a review". World Journal of Surgical Oncology 16.1 (2018): 63-76.

Volume 2 Issue 2 February 2022

(C) All rights are reserved by Samuel I Ogenyi., et al. 